PROCEEDINGS OF THE

AMERICAN MATHEMATICAL SOCIETY

Volume 136, Number 6, June 2008, Pages 1945-1953

S 0002-9939(08)09151-X

Article electronically published on February 14, 2008

\title{
ON EXCEPTIONAL EIGENVALUES OF THE LAPLACIAN FOR $\Gamma_{0}(N)$
}

\author{
XIAN-JIN LI
}

(Communicated by Wen-Ching Winnie Li)

\begin{abstract}
An explicit Dirichlet series is obtained, which represents an analytic function of $s$ in the half-plane $\Re s>1 / 2$ except for having simple poles at points $s_{j}$ that correspond to exceptional eigenvalues $\lambda_{j}$ of the nonEuclidean Laplacian for Hecke congruence subgroups $\Gamma_{0}(N)$ by the relation $\lambda_{j}=s_{j}\left(1-s_{j}\right)$ for $j=1,2, \cdots, S$. Coefficients of the Dirichlet series involve all class numbers $h_{d}$ of real quadratic number fields. But, only the terms with $h_{d} \gg d^{1 / 2-\epsilon}$ for sufficiently large discriminants $d$ contribute to the residues $m_{j} / 2$ of the Dirichlet series at the poles $s_{j}$, where $m_{j}$ is the multiplicity of the eigenvalue $\lambda_{j}$ for $j=1,2, \cdots, S$. This may indicate (I'm not able to prove yet) that the multiplicity of exceptional eigenvalues can be arbitrarily large. On the other hand, by density theorem the multiplicity of exceptional eigenvalues is bounded above by a constant depending only on $N$.
\end{abstract}

\section{INTRODUCTION}

Let $N$ be a positive integer. Denote by $\Gamma_{0}(N)$ the Hecke congruence subgroup of level $N$. The non-Euclidean Laplacian $\Delta$ on the upper half-plane $\mathcal{H}$ is given by

$$
\Delta=-y^{2}\left(\frac{\partial^{2}}{\partial x^{2}}+\frac{\partial^{2}}{\partial y^{2}}\right) \text {. }
$$

Let $D$ be the fundamental domain of $\Gamma_{0}(N)$. Eigenfunctions of the discrete spectrum of $\Delta$ are nonzero real-analytic solutions of the equation $\Delta \psi=\lambda \psi$ such that $\psi(\gamma z)=\psi(z)$ for all $\gamma$ in $\Gamma_{0}(N)$ and such that $\psi$ is square integrable on $D$ with respect to the Poincaré measure $d z$ of the upper half-plane.

The Hecke operators $T_{n}, n=1,2, \cdots,(n, N)=1$, which act in the space of automorphic functions with respect to $\Gamma_{0}(N)$, are defined by

$$
\left(T_{n} f\right)(z)=\frac{1}{\sqrt{n}} \sum_{a d=n, 0 \leq b<d} f\left(\frac{a z+b}{d}\right) .
$$

It is well-known (see Iwaniec [3]) that there exists a maximal orthonormal system of eigenfunctions of $\Delta$ such that each of them is an eigenfunction of all the Hecke operators. Let $\lambda_{j}, j=1,2, \cdots$, be an enumeration in increasing order of all positive discrete eigenvalues of $\Delta$ for $\Gamma_{0}(N)$ with an eigenvalue of multiplicity $m$ appearing

Received by the editors May 15, 2006 and, in revised form, March 5, 2007.

2000 Mathematics Subject Classification. Primary 11F37, 11F72.

Key words and phrases. Class numbers, Hecke operators, Maass wave forms, real quadratic fields.

This research was supported by National Security Agency H98230-06-1-0061.

(C)2008 American Mathematical Society 1945

Reverts to public domain 28 years from publication 
$m$ times, and let $\kappa_{j}=\sqrt{\lambda_{j}-1 / 4}$ with $\Im \kappa_{j}>0$ if $\lambda_{j}<1 / 4$. We denote the multiplicity of the eigenvalue $\lambda_{j}$ by $m_{j}$ for $j=1,2, \cdots$.

If $\lambda$ is a positive discrete eigenvalue less than $1 / 4$, we call it an exceptional eigenvalue. Let $\lambda_{1}, \cdots, \lambda_{S}$ be exceptional eigenvalues of the Laplacian $\Delta$ for $\Gamma_{0}(N)$.

In 1965, A. Selberg [10] made the following fundamental conjecture.

Selberg's eigenvalue conjecture. If $\lambda$ is a nonzero discrete eigenvalue of the non-Euclidean Laplacian for any congruence subgroup, then $\lambda \geq 1 / 4$.

A. Selberg [10] proved that $\lambda \geqslant 3 / 16$. The best available lower bound $\lambda \geq$ $975 / 4096$ is due to Kim and Sarnak [4. It was obtained by combining automorphic lifts $\mathrm{sym}^{3}: G L(2) \rightarrow G L(4)\left[5\right.$ and $\mathrm{sym}^{4}: G L(2) \rightarrow G L(5)$ [4 with families of $L$-functions 8 . We note that if the general functorial conjectures concerning the automorphic lifts sym $\mathrm{sym}^{k}: G L(2) \rightarrow G L(k+1)$ are true for all $k>1$, then Selberg's eigenvalue conjecture would follow.

Let $\Omega$ be the set of all the positive integers $d$ such that $d \equiv 0$ or $1(\bmod 4)$ and such that $d$ is not a square of an integer. In this paper, we indicate an elementary approach towards the Selberg eigenvalue conjecture. Namely, we prove the following theorem.

Theorem 1. Let

$$
L(s)=\sum_{k \mid N} \sum_{d \in \Omega, k \mid u_{d}} \prod_{p^{2 l} \mid(d, N / k)} p^{l} \prod_{p \mid N / k}\left(1+\left(\frac{d}{p}\right)\right) \frac{h_{d k^{2}} \ln \epsilon_{d k^{2}}}{\left(d u_{d}^{2}\right)^{s}}-\sum_{d \in \Omega} \frac{h_{d} \ln \epsilon_{d}}{\left(d u_{d}^{2}\right)^{s}}
$$

where $\left(v_{d}, u_{d}\right)$ is the smallest positive solution of Pell's equation $v^{2}-d u^{2}=4$ and the product on $p^{2 l}$ is over all distinct primes $p$ with $p^{2 l}$ being the greatest even $p$ power factor of $(d, N / k)$. Then $L(s)$ represents an analytic function of $s$ in the half-plane $\Re s>1 / 2$ except for having simple poles at $s_{j}=\frac{1}{2}-i \kappa_{j}, j=1,2, \cdots, S$. Moreover, we have

$$
m_{j}=2 \operatorname{Res}_{s=s_{j}} L(s)
$$

for $j=1,2, \cdots, S$.

Corollary 2. If $N$ is square free, then the series

$$
L_{1}(s)=\sum_{\substack{m|N, k| N \\(m, k) \neq(1,1)}} k \frac{\mu((m, k))}{(m, k)} \sum_{d \in \Omega, k \mid u_{d}}\left(\frac{d}{m}\right) \frac{h_{d} \ln \epsilon_{d}}{\left(d u_{d}^{2}\right)^{s}}
$$

represents an analytic function of $s$ in the half-plane $\Re s>1 / 2$ except for having simple poles at $s_{j}=\frac{1}{2}-i \kappa_{j}, j=1,2, \cdots, S$, where $\left(v_{d}, u_{d}\right)$ is the smallest positive solution of Pell's equation $v^{2}-d u^{2}=4$. Moreover, we have

$$
m_{j}=2 \operatorname{Res}_{s=s_{j}} L_{1}(s) .
$$

This work was initiated while the author attended the workshop on Eisenstein Series and Applications at the American Institute of Mathematics (AIM), August 1519, 2005. He wants to thank AIM for the invitation to attend the workshop. The author is grateful to the referee for pointing out two inaccuracies in the original version of the manuscript and for the suggestion of improving the presentation of the paper. 


\section{Proofs of Theorem 1 and Corollary 2}

We denote by $h_{d}$ the class number of indefinite rational quadratic forms with discriminant $d$. Let

$$
\epsilon_{d}=\frac{v_{0}+u_{0} \sqrt{d}}{2},
$$

where the pair $\left(v_{0}, u_{0}\right)$ is the smallest positive solution of Pell's equation $v^{2}-d u^{2}=$ 4.

Lemma 2.1. Let $d$ and $d_{1}$ be integers in $\Omega$. If $d_{1}=d l^{2}$, then

$$
h_{d_{1}} \ln \epsilon_{d_{1}}=l \prod_{p \mid l}\left(1-\left(\frac{d}{p}\right) p^{-1}\right) h_{d} \ln \epsilon_{d} .
$$

Proof. The stated identity follows from Dirichlet's class number formula (see, $\S 100$ of Dirichlet [2])

$$
h_{d_{1}} \ln \epsilon_{d_{1}}=\sqrt{d_{1}} L\left(1, \chi_{d_{1}}\right)
$$

and the identity

$$
L\left(1, \chi_{d_{1}}\right)=L\left(1, \chi_{d}\right) \prod_{p \mid l}\left(1-\left(\frac{d}{p}\right) p^{-1}\right)
$$

Lemma 2.2. Let $d$ and $d_{1}$ be integers in $\Omega$, and let $d_{1}=d l^{2}$. Then $\epsilon_{d_{1}}=\epsilon_{d}^{\nu_{l}}$ for a positive integer $\nu_{l}$.

Proof. If $\left(v_{1}, u_{1}\right)$ is the smallest positive solution of Pell's equation

$$
v^{2}-d l^{2} u^{2}=4
$$

then

$$
\epsilon_{d_{1}}=\frac{v_{1}+\sqrt{d_{1}} u_{1}}{2}
$$

Let $\left(v_{0}, u_{0}\right)$ be the smallest positive solution of Pell's equation

$$
v^{2}-d u^{2}=4 .
$$

By $\S 85$ of Dirichlet 22, all positive solutions $(v, u)$ of $(2.2)$ are given by the formula

$$
\frac{v+\sqrt{d} u}{2}=\left(\frac{v_{0}+\sqrt{d} u_{0}}{2}\right)^{n}
$$

for positive integers $n$. Since $\left(v_{1}, l u_{1}\right)$ is a positive solution of $(2.2)$, there exists a positive integer $\nu_{l}$ such that

$$
\frac{v_{1}+\sqrt{d_{1}} u_{1}}{2}=\left(\frac{v_{0}+\sqrt{d} u_{0}}{2}\right)^{\nu_{l}}
$$

Lemma 2.3. Let $N$ be any positive integer, and let

$$
L_{N}(s)=\sum_{k \mid N} k^{1-2 s} \sum_{d \in \Omega} \sum_{u} \prod_{p^{2 l} \mid(d, N / k)} p^{l} \prod_{p \mid N / k}\left(1+\left(\frac{d}{p}\right)\right) \prod_{p \mid k}\left(1-\left(\frac{d}{p}\right) p^{-1}\right) \frac{h_{d} \ln \epsilon_{d}}{\left(d u^{2}\right)^{s}}
$$


for $\Re s>1$, where the sum on $u$ is over all positive integers $u$ such that $\sqrt{4+d k^{2} u^{2}} \in$ $\mathbb{Z}$ and where the product on $p^{2 l}$ is over all distinct primes $p$ with $p^{2 l}$ being the greatest even p-power factor of $(d, N / k)$. Then $L_{N}(s)$ is analytic for $\Re s>1$ and has analytic continuation to the half-plane $\Re s>1 / 2$ except for having simple poles at $s=1$ and $s_{j}=\frac{1}{2}-i \kappa_{j}, j=1,2, \cdots, S$. Moreover, we have

$$
m_{j}=2 \operatorname{Res}_{s=s_{j}} L_{N}(s)
$$

for $j=1,2, \cdots, S$.

Proof. Let

$$
h(r)=4^{s} \sqrt{\pi} \frac{\Gamma(s-1 / 2)}{\Gamma(s)} \int_{0}^{\infty}\left(u+\frac{1}{u}+2\right)^{1 / 2-s} u^{i r-1} d u
$$

for $\Re s>1 / 2$. Then the lemma follows from Theorem 4.3, the proof of Lemma 5.3, the proof of Theorem 1 in $\mathrm{Li}$ [7, and the Selberg trace formula

$$
\begin{aligned}
h(-i / 2) & +\sum_{j=1}^{\infty} h\left(\kappa_{j}\right) m_{j} \\
& =4^{1 / 2+s} \sqrt{\pi} \frac{\Gamma(s-1 / 2)}{\Gamma(s)} L_{N}(s)+f(s)
\end{aligned}
$$

for $\Re s>1$ where $f(s)$ is a certain analytic function of $s$ in the half-plane $\Re s \geq 1 / 2$ except for a possible pole at $s=1 / 2$ (see (4.4) of Li [7]).

Remark 2.4. Siegel [1] proved that

$$
\lim _{d \rightarrow \infty} \frac{\ln \left(h_{d} \ln \epsilon_{d}\right)}{\ln d}=\frac{1}{2} .
$$

Lemma 2.5. Let

$$
\bar{L}_{N}(s)=\sum_{k \mid N} \sum_{d \in \Omega, k \mid u_{d}} \prod_{p^{2 l} \mid(d, N / k)} p^{l} \prod_{p \mid N / k}\left(1+\left(\frac{d}{p}\right)\right) \frac{h_{d k^{2}} \ln \epsilon_{d k^{2}}}{\left(d u_{d}^{2}\right)^{s}}
$$

for $\Re s>1$, where $\left(v_{d}, u_{d}\right)$ is the smallest positive solution of Pell's equation $v^{2}-$ $d u^{2}=4$ and the product on $p^{2 l}$ is over all distinct primes $p$ with $p^{2 l}$ being the greatest even $p$-power factor of $(d, N / k)$. Then $\bar{L}_{N}(s)$ is analytic for $\Re s>1$ and has analytic continuation to the half-plane $\Re s>1 / 2$ except for having simple poles at $s=1$ and $s_{j}=\frac{1}{2}-i \kappa_{j}, j=1,2, \cdots, S$. Moreover, we have

$$
m_{j}=2 \operatorname{Res}_{s=s_{j}} \bar{L}_{N}(s) \text {. }
$$

Proof. By Lemma 2.3, the function

$$
L_{N}(s)=\sum_{k \mid N} k^{1-2 s} \sum_{d \in \Omega} \sum_{u} \prod_{p^{2 l}} \prod_{\mid(d, N / k)} p^{l} \prod_{p \mid N / k}\left(1+\left(\frac{d}{p}\right)\right) \prod_{p \mid k}\left(1-\left(\frac{d}{p}\right) p^{-1}\right) \frac{h_{d} \ln \epsilon_{d}}{\left(d u^{2}\right)^{s}}
$$

has analytic continuation to the half-plane $\Re s>1 / 2$ except for having simple poles at $s=1$ and $s_{j}=\frac{1}{2}-i \kappa_{j}, j=1,2, \cdots, S$, where the sum on $u$ is over all positive solutions of Pell's equation

$$
v^{2}-d k^{2} u^{2}=4
$$


Let $\left(v_{k}, u_{k}\right)$ be the smallest positive solution of (2.5). By $\S 85$ of Dirichlet [2], all positive solutions $(v, u)$ of $(2.5)$ are given by the formula

$$
\frac{v+\sqrt{d} k u}{2}=\left(\frac{v_{k}+\sqrt{d} k u_{k}}{2}\right)^{n}
$$

for $n=1,2, \cdots$. Hence, we have

$$
\begin{aligned}
\sqrt{d} k u & =\left(\frac{v_{k}+\sqrt{d} k u_{k}}{2}\right)^{n}\left(1-\left(\frac{v_{k}+\sqrt{d} k u_{k}}{2}\right)^{-2 n}\right) \\
& >\left(\frac{v_{k}+\sqrt{d} k u_{k}}{2}\right)^{n}(1+2 / \sqrt{d} k)^{-1} .
\end{aligned}
$$

Let $\sigma=\Re s>1 / 2$, and let $\tau(n)$ be the number of positive divisors of an integer $n$. By (2.6) and (2.3), we have

$$
\begin{aligned}
& \left|\sum_{k \mid N} k^{1-2 s} \sum_{d \in \Omega} \sum_{u \neq u_{k}} \prod_{p^{2 l} \mid(d, N / k)} p^{l} \prod_{p \mid N / k}\left(1+\left(\frac{d}{p}\right)\right) \prod_{p \mid k}\left(1-\left(\frac{d}{p}\right) p^{-1}\right) \frac{h_{d} \ln \epsilon_{d}}{\left(d u^{2}\right)^{s}}\right| \\
& \leq \sum_{k \mid N} \sqrt{k N} 2^{\tau(N)} \sum_{d \in \Omega}(1+2 / \sqrt{d} k)^{2 \sigma} h_{d} \ln \epsilon_{d} \sum_{n=2}^{\infty}\left(\frac{v_{k}+\sqrt{d} k u_{k}}{2}\right)^{-2 n \sigma} \\
& \leq \sum_{k \mid N} \sqrt{k N} 2^{\tau(N)} 3^{2 \sigma+1} \sum_{d \in \Omega} h_{d} \ln \epsilon_{d}\left(\frac{v_{k}+\sqrt{d} k u_{k}}{2}\right)^{-4 \sigma} \\
& \leq 16^{\sigma} N 2^{\tau(N)} 3^{2 \sigma+1} \sum_{k \mid N} \sum_{d \in \Omega} d^{1 / 2+\epsilon-2 \sigma}\left(k u_{k}\right)^{-4 \sigma} .
\end{aligned}
$$

Note that $\tau(n) \ll n^{\epsilon}$ as $n \rightarrow \infty$. Since, for a fixed positive integer $v$, there are at most $\tau\left(v^{2}-4\right)$ number of $d$ 's in $\Omega$ such that $v^{2}-d u^{2}=4$ for positive integers $u$, we have

$$
\sum_{d \in \Omega} d^{1 / 2+\epsilon-2 \sigma}\left(k u_{k}\right)^{-4 \sigma} \leq \sum_{d \in \Omega}\left(v_{k}^{2}-4\right)^{-\sigma} \leq \sum_{v=3}^{\infty} \frac{\tau\left(v^{2}-4\right)}{\left(v^{2}-4\right)^{\sigma}} \ll \sum_{v=3}^{\infty} \frac{1}{\left(v^{2}-4\right)^{\sigma-\epsilon}}<\infty
$$

for $\sigma>1 / 2$. Hence, the series

$$
\sum_{k \mid N} k^{1-2 s} \sum_{d \in \Omega} \sum_{u \neq u_{k}} \prod_{p^{2 l} \mid(d, N / k)} p^{l} \prod_{p \mid N / k}\left(1+\left(\frac{d}{p}\right)\right) \prod_{p \mid k}\left(1-\left(\frac{d}{p}\right) p^{-1}\right) \frac{h_{d} \ln \epsilon_{d}}{\left(d u^{2}\right)^{s}},
$$

represents an analytic function of $s$ in the half-plane $\Re s>1 / 2$. It follows from (2.4) that the function

$$
\sum_{k \mid N} k^{1-2 s} \sum_{d \in \Omega} \prod_{p^{2 l} \mid(d, N / k)} p^{l} \prod_{p \mid N / k}\left(1+\left(\frac{d}{p}\right)\right) \prod_{p \mid k}\left(1-\left(\frac{d}{p}\right) p^{-1}\right) \frac{h_{d} \ln \epsilon_{d}}{\left(d u_{k}^{2}\right)^{s}}
$$

has analytic continuation to the half-plane $\Re s>1 / 2$ except for having simple poles at $s=1$ and $s_{j}=\frac{1}{2}-i \kappa_{j}, j=1,2, \cdots, S$.

Next, let $\left(v_{0}, u_{0}\right)$ be the smallest positive solution of Pell's equation

$$
v^{2}-d u^{2}=4 .
$$


Let $k$ be a divisor of $N$. If $\left(v_{k}, k u_{k}\right)$ is a solution of $(2.8)$ different from $\left(v_{0}, u_{0}\right)$, then by Lemma 2.2 there exists an integer $n \geq 2$ such that

$$
\frac{v_{k}+\sqrt{d} k u_{k}}{2}=\left(\frac{v_{0}+\sqrt{d} u_{0}}{2}\right)^{n} \text {. }
$$

Hence, we have

$$
\begin{aligned}
\sqrt{d} k u_{k}= & \left(\frac{v_{0}+\sqrt{d} u_{0}}{2}\right)^{n}\left(1-\left(\frac{v_{0}+\sqrt{d} u_{0}}{2}\right)^{-2 n}\right) \\
& >\left(\frac{v_{0}+\sqrt{d} u_{0}}{2}\right)^{n}(1+2 / \sqrt{d})^{-1} .
\end{aligned}
$$

By (2.9) and (2.3), we have

$$
\begin{aligned}
& \left|\sum_{k \mid N} k^{1-2 s} \sum_{d \in \Omega, k u_{k} \neq u_{0}} \prod_{p^{2 l} \mid(d, N / k)} p^{l} \prod_{p \mid N / k}\left(1+\left(\frac{d}{p}\right)\right) \prod_{p \mid k}\left(1-\left(\frac{d}{p}\right) p^{-1}\right) \frac{h_{d} \ln \epsilon_{d}}{\left(d u_{k}^{2}\right)^{s}}\right| \\
& \leq \sum_{k \mid N} \sqrt{k N} 2^{\tau(N)} \sum_{d \in \Omega, k u_{k} \neq u_{0}}(1+2 / \sqrt{d})^{2 \sigma} h_{d} \ln \epsilon_{d}\left(\frac{v_{0}+\sqrt{d} u_{0}}{2}\right)^{-2 n \sigma} \\
& \leq \sum_{k \mid N} \sqrt{k N} 2^{\tau(N)} 9^{\sigma} \sum_{d \in \Omega, k u_{k} \neq u_{0}} h_{d} \ln \epsilon_{d}\left(\frac{v_{0}+\sqrt{d} u_{0}}{2}\right)^{-4 \sigma} \\
& \leq 16^{\sigma} \tau(N) N 2^{\tau(N)} 9^{\sigma} \sum_{d \in \Omega} d^{1 / 2+\epsilon-2 \sigma} u_{0}^{-4 \sigma} .
\end{aligned}
$$

Since

$$
\sum_{d \in \Omega} d^{1 / 2+\epsilon-2 \sigma} u_{0}^{-4 \sigma} \leq \sum_{d \in \Omega}\left(v_{0}^{2}-4\right)^{-\sigma} \leq \sum_{v=3}^{\infty} \frac{\tau\left(v^{2}-4\right)}{\left(v^{2}-4\right)^{\sigma}} \ll \sum_{v=3}^{\infty} \frac{1}{\left(v^{2}-4\right)^{\sigma-\epsilon}}<\infty
$$

for $\sigma>1 / 2$, the series

$$
\sum_{k \mid N} k^{1-2 s} \sum_{d \in \Omega, k u_{k} \neq u_{0}} \prod_{p^{2 l} \mid(d, N / k)} p^{l} \prod_{p \mid N / k}\left(1+\left(\frac{d}{p}\right)\right) \prod_{p \mid k}\left(1-\left(\frac{d}{p}\right) p^{-1}\right) \frac{h_{d} \ln \epsilon_{d}}{\left(d u_{k}^{2}\right)^{s}}
$$

represents an analytic function of $s$ in the half-plane $\Re s>1 / 2$. It follows from (2.7) that the function

$$
\sum_{k \mid N} k \sum_{d \in \Omega, k \mid u_{0}} \prod_{p^{2 l} \mid(d, N / k)} p^{l} \prod_{p \mid N / k}\left(1+\left(\frac{d}{p}\right)\right) \prod_{p \mid k}\left(1-\left(\frac{d}{p}\right) p^{-1}\right) \frac{h_{d} \ln \epsilon_{d}}{\left(d u_{0}^{2}\right)^{s}}
$$

has analytic continuation to the half-plane $\Re s>1 / 2$ except for having simple poles at $s=1$ and $s_{j}=\frac{1}{2}-i \kappa_{j}, j=1,2, \cdots, S$, where the product on $p^{2 l}$ is over all distinct primes $p$ with $p^{2 l}$ being the greatest even $p$-power factor of $(d, N / k)$. By Lemma 2.1 we can write $(2.10)$ as

$$
\sum_{k \mid N} \sum_{d \in \Omega, k \mid u_{0}} \prod_{p^{2 l} \mid(d, N / k)} p^{l} \prod_{p \mid N / k}\left(1+\left(\frac{d}{p}\right)\right) \frac{h_{d k^{2}} \ln \epsilon_{d k^{2}}}{\left(d u_{0}^{2}\right)^{s}} .
$$

This completes the proof of the lemma. 
Proof of Theorem 1. It is proved in [6] that the series

$$
F(s)=\sum_{d \in \Omega} \frac{h_{d} \ln \epsilon_{d}}{d^{s}} \sum_{\substack{u>0 \\ v^{2}-d u^{2}=4}} \frac{1}{u^{2 s}}
$$

represents an analytic function of $s$ in the half-plane $\Re s>1 / 2$ except for having a simple poles at $s=1$. By (2.3), (3.4), (3.5), Lemma 3.5, Lemma 4.1, and Lemma 4.2 of [6], we have that

$$
F(s)-h(-i / 2)
$$

is analytic in the half-plane $\Re s>1 / 2$. Let $\left(v_{d}, u_{d}\right)$ be the smallest positive solution of Pell's equation $v^{2}-d u^{2}=4$. If $u \neq u_{d}$, then

$$
\frac{v+\sqrt{d} u}{2}=\left(\frac{v_{d}+\sqrt{d} u_{d}}{2}\right)^{\nu}
$$

for some positive integer $\nu \geq 2$. Similarly as in (2.9), we can obtain that

$$
\sqrt{d} u \geq \frac{1}{3} \epsilon_{d}^{\nu}
$$

It follows that

$$
\begin{aligned}
\left|\sum_{d \in \Omega} \frac{h_{d} \ln \epsilon_{d}}{d^{s}} \sum_{\substack{u \neq u_{d} \\
v^{2}-d u^{2}=4}} \frac{1}{u^{2 s}}\right| & \leq 9 \sum_{d \in \Omega} \frac{h_{d} \ln \epsilon_{d}}{\epsilon_{d}^{4 \sigma}} \\
& \leq 2^{4 \sigma} 9 \sum_{d \in \Omega} d^{1 / 2+\epsilon-2 \sigma} u_{d}^{-4 \sigma}<\infty
\end{aligned}
$$

for $\sigma=\Re s>1 / 2$. Let

$$
l(s)=\sum_{d \in \Omega} \frac{h_{d} \ln \epsilon_{d}}{\left(d u_{d}^{2}\right)^{s}} .
$$

By (2.11) and (2.12), we obtain that

$$
l(s)-h(-i / 2)
$$

is analytic in the half-plane $\Re s>1 / 2$.

Let $\bar{L}_{N}(s)$ be given as in Lemma 2.5. Then by (1.4), (4.4), (4.5), Theorem 4.3, Lemma 5.1, and Lemma 5.3 of [7, we have that

$$
\bar{L}_{N}(s)-h(-i / 2)
$$

is an analytic function of $s$ in the half-plane $\Re s>1 / 2$ except for simple poles at $s=1 / 2-i \kappa_{j}, j=1,2, \cdots, S$. It follows from (2.13) and (2.14) that

$$
L(s)=\bar{L}_{N}(s)-l(s)
$$

represents an analytic function of $s$ in the half-plane $\Re s>1 / 2$ except for simple poles at $s_{j}=1 / 2-i \kappa_{j}, j=1,2, \cdots, S$. Moreover, we have

$$
m_{j}=2 \operatorname{Res}_{s=s_{j}} L(s)
$$

for $j=1,2, \cdots, S$.

This completes the proof of the theorem. 
Proof of Corollary 2. By Theorem 1 the series

$$
\sum_{k \mid N} k \sum_{d \in \Omega, k \mid u_{d}} \prod_{p^{2 l} \mid(d, N / k)} p^{l} \prod_{p \mid N / k}\left\{1+\left(\frac{d}{p}\right)\right\} \prod_{p \mid k}\left\{1-\frac{1}{p}\left(\frac{d}{p}\right)\right\} \frac{h_{d} \ln \epsilon_{d}}{\left(d u_{d}^{2}\right)^{s}}-\sum_{d \in \Omega} \frac{h_{d} \ln \epsilon_{d}}{\left(d u_{d}^{2}\right)^{s}}
$$

represents an analytic function of $s$ in the half-plane $\Re s>1 / 2$ except for having simple poles at $s_{j}=\frac{1}{2}-i \kappa_{j}, j=1,2, \cdots, S$, where $\left(v_{d}, u_{d}\right)$ is the smallest positive solution of Pell's equation $v^{2}-d u^{2}=4$ and the product on $p^{2 l}$ is over all distinct primes $p$ with $p^{2 l}$ being the greatest even $p$-power factor of $(d, N / k)$. Moreover, we have

for $j=1,2, \cdots, S$.

$$
m_{j}=2 \operatorname{Res}_{s=s_{j}} L(s)
$$

Since $N$ is square free, we have

$$
\prod_{p \mid N / k}\left\{1+\left(\frac{d}{p}\right)\right\} \prod_{p \mid k}\left\{1-\frac{1}{p}\left(\frac{d}{p}\right)\right\}=\sum_{m \mid N} \frac{\mu((m, k))}{(m, k)}\left(\frac{d}{m}\right)
$$

where $\mu(n)$ is the Möbius function and $(m, k)$ denotes the greatest common divisor of $m$ and $k$. By using the identity (2.15), we can write

$$
\begin{aligned}
& \sum_{k \mid N} k \sum_{d \in \Omega, k \mid u_{d}} \prod_{p^{2 l} \mid(d, N / k)} p^{l} \prod_{p \mid N / k}\left\{1+\left(\frac{d}{p}\right)\right\} \prod_{p \mid k}\left\{1-\frac{1}{p}\left(\frac{d}{p}\right)\right\} \frac{h_{d} \ln \epsilon_{d}}{\left(d u_{d}^{2}\right)^{s}}-\sum_{d \in \Omega} \frac{h_{d} \ln \epsilon_{d}}{\left(d u_{d}^{2}\right)^{s}} \\
& =\sum_{m|N, k| N} k \frac{\mu((m, k))}{(m, k)} \sum_{d \in \Omega, k \mid u_{d}}\left(\frac{d}{m}\right) \frac{h_{d} \ln \epsilon_{d}}{\left(d u_{d}^{2}\right)^{s}}-\sum_{d \in \Omega} \frac{h_{d} \ln \epsilon_{d}}{\left(d u_{d}^{2}\right)^{s}} \\
& =\sum_{\substack{m|N, k| N \\
(m, k) \neq(1,1)}} k \frac{\mu((m, k))}{(m, k)} \sum_{d \in \Omega, k \mid u_{d}}\left(\frac{d}{m}\right) \frac{h_{d} \ln \epsilon_{d}}{\left(d u_{d}^{2}\right)^{s}} .
\end{aligned}
$$

It then follows that the series

$$
L_{1}(s)=\sum_{\substack{m|N, k| N \\(m, k) \neq(1,1)}} k \frac{\mu((m, k))}{(m, k)} \sum_{d \in \Omega, k \mid u_{d}}\left(\frac{d}{m}\right) \frac{h_{d} \ln \epsilon_{d}}{\left(d u_{d}^{2}\right)^{s}}
$$

represents an analytic function of $s$ in the half-plane $\Re s>1 / 2$ except for having simple poles at those points $s_{j}=\frac{1}{2}-i \kappa_{j}, j=1,2, \cdots, S$, where $\left(v_{d}, u_{d}\right)$ is the smallest positive solution of Pell's equation $v^{2}-d u^{2}=4$. Moreover, we have

$$
m_{j}=2 \operatorname{Res}_{s=s_{j}} L_{1}(s)
$$

for $j=1,2, \cdots, S$.

This completes the proof of the corollary.

\section{REFERENCES}

1. J. B. Conrey and Xian-Jin Li, On the trace of Hecke operators for Maass forms for congruence subgroups, Forum Math. 13 (2001), 447-484. MR.1830242 (2002e:11057)

2. P. G. L. Dirichlet, Lectures on Number Theory, Amer. Math. Soc., Providence, R. I., 1999. MR:1710911 (2000e:01045)

3. H. Iwaniec, Spectral Methods of Automorphic Forms, Amer. Math. Soc., Providence, 2002. MR.1942691 (2003k:11085)

4. H. Kim, Functoriality for the exterior square of $G L(4)$ and the symmetric fourth of $G L(2)$. With the appendix: Refined estimates towards the Ramanujan and Selberg conjectures (by H. Kim and P. Sarnak), J. Amer. Math. Soc. 16 (2003), 139-183. MR1937203(2003k:11083) 
5. H. Kim and F. Shahidi, Functorial products for $G L(2) \times G L(3)$ and the symmetric cube for GL(2), Ann. of Math. 155 (2002), 837-893. MR.1923967 (2003m:11075)

6. Xian-Jin Li, On the trace of Hecke operators for Maass forms, CRM Proc. and Lecture Notes, Vol. 19, Amer. Math. Soc., Providence, R. I. (1999), 215-229. MR1684604 (2000b:11050)

7. Xian-Jin Li, On the trace of Hecke operators for Maass forms for congruence subgroups. II, Forum Math. 17 (2005), 1-30. MR2110536 (2005i:11059)

8. W. Luo, Z. Rudnick and P. Sarnak, On Selberg's eigenvalue conjecture, Geom. Funct. Anal. 5 (1995), 387-401. MR1334872 (96h:11045)

9. P. Sarnak, Class numbers of indefinite binary quadratic forms II, J. Number Theory 21 (1985), 333-346. MR814010 (87h:11027)

10. A. Selberg, On the estimation of Fourier coefficients of modular forms, in "Atle Selberg Collected Papers," Vol. I, Springer-Verlag, 1989, pp. 506-520. MR0182610 (32:93)

11. C. L. Siegel, Über die Classenzahl quadratischer Zahlkörper, Acta Arith. 1 (1935), 83-86.

Department of Mathematics, Brigham Young University, Provo, Utah 84602

E-mail address: xianjin@math.byu.edu 